\title{
Study the Flux and Energy of the Helium lons Beam Produced by Two Different Dense Plasma Focus Devices When Gas Pressure Changes
}

\author{
Walid Sahyouni ${ }^{1}$, Alaa Nassif ${ }^{2}$, Ola Zeidan ${ }^{1}$ \\ ${ }^{1}$ Plasma Physics Research Unit, Department of Physics, Faculty of Science, Al-Baath University, Homs, Syria \\ ${ }^{2}$ Faculty of Engineering, Al-Wataniya Private University, Hama, Syria
}

\section{Email address:}

wsahyouni@albaath-univ.edu.sy (W. Sahyouni), Alaa.nassif@wpu.edu.sy (A. Nassif), ozedan@albaath-univ.edu.sy (O. Zeidan)

\section{To cite this article:}

Walid Sahyouni, Alaa Nassif, Ola Zeidan. Study the Flux and Energy of the Helium Ions Beam Produced by Two Different Dense Plasma Focus Devices When Gas Pressure Changes. American Journal of Mechanics and Applications. Vol. 8, No. 1, 2020 pp. $16-20$. doi: 10.11648/j.ajma.20200801.13

Received: September 25, 2019; Accepted: October 7, 2019; Published: January 10, 2020

\begin{abstract}
In this study, the flux and energy of the helium ions beam produced by United Nations University/International Centre for Theoretical Physics Plasma Fusion Facility (UNU/ICTP PFF) and NX2 dense plasma focus devices, were studied when the pressure of gas changed by carrying out numerical experiments using Lee code. The results showed that the value of the ions beam flux increased with increasing the gas pressure until reaching a maximum value $1.463 \mathrm{E}+28$ ions. m-2s- 1 at 7 Torr in UNU/ ICTP PFF device while in NX2 device the maximum value is $6.442 \mathrm{E}+27$ ions. $\mathrm{m}-2 \mathrm{~s}-1$ at 14 Torr and then decreasing while continuing pressure increasing, but the energy of ions beam showed the opposite behavior where the maximum value of energy of the helium ions beam was 13 Joul at 5 Torr in UNU/ICTP PFF device while it was 144 Joul at 14 Torr in NX2 device this is due to the effect of the geometric dimensions on the value of the induced voltage within the plasma pinch.
\end{abstract}

Keywords: Plasma Focus, Lee Code, Plasma Pinch

\section{Introduction}

When matter is heated to a high enough temperature, it ionizes and becomes plasma. It emits electromagnetic radiation. The spectrum depends on the temperature and the material. The higher the temperature and the denser the matter, the more intense is the radiation. Beams of electrons and ions may also be emitted. Typically the temperatures are above several million $\mathrm{K}$ and compressed densities above atmospheric density starting with a gas a hundredth of an atmospheric density. One way of achieving such highly heated material is by means of an electrical discharge through gases. As the gas is heated, it expands, lowering the density and making it difficult to heat further. Thus it is necessary to compress the gas whilst heating it, in order to achieve sufficiently intense conditions. An electrical discharge between two electrodes produces an azimuthal magnetic field which interacts with the column of current, giving rise to a self-compression force which tends to constrict (or pinch) the column. In order to 'pinch', or hold together, a column of gas at about atmospheric density at a temperature of 1 million $\mathrm{K}$, a rather large pressure has to be exerted by the pinching magnetic field. Thus an electric current of at least hundreds of $\mathrm{kA}$ is required even for a column of small radius of say $1 \mathrm{~mm}$. Moreover, the dynamic process requires that the current rises very rapidly, typically in under $0.1 \mu \mathrm{s}$ in order to have a sufficiently hot and dense pinch. Such a pinch is known as a super fast super dense pinch, and requires special MA fastrise (ns) pulsed-lines. These lines may be powered by capacitor banks, and suffer the disadvantage of conversion losses and high cost due to the cost of the high technology pulse-shaping line, in addition to the capacitor banks. [1]

The Lee model code [2, 3] uses the snowplow model [4] in the axial phase, the slug model [5] with 'communication delay' and thermodynamics in the early radial phase and a radiationcoupled compression in the pinch phase; all the phases being rigorously circuit-coupled so as to be energy and charge consistent. The code has on the one hand been successful in developing a number of deep insights and on the other hand has been successful in applications ranging from correlation with experiments in dynamics, neutron and soft X-ray (SXR) yields, 
in fast ion beams (FIB) and fast plasma streams (FPS) properties and in designing plasma focus and variants. Its success on so many fronts appears to be due to its use of 4 parameters (fitted to a measured current waveform) which in one sweep incorporates all the mechanisms and effects occurring in the plasma focus including mechanisms and effects difficult to compute or even as yet unknown [6].

The ions beam emitted from dense plasma focus devices studied widely to use it in material science applications and production short lived radioisotopes [7-12].

In this study we used Lee code to carrying out many numerical experiments to study the variations of flux and energy of ions beam emitted from two dense plasma focus devices when the pressure of helium gas was changed and explain these differences.

\section{Flux and Energy of Ions Beam Emitted from Plasma Focus Pinch}

Lee and Saw studied the properties of the ions beam from the collapse of the plasma pinch and concluded the relationship of flux and ion beam energy as follows [13, 14]:

Flux (ions. $\mathrm{m}^{-2} \cdot \mathrm{s}^{-1}$ ):

$$
F L U X=2.75 \times 10^{15} \frac{f_{e}}{\sqrt{M . Z_{e f f}}} \cdot \frac{\ln \left[b / r_{P}\right]}{r_{P}^{2}} \cdot \frac{I_{\text {Pinch }}^{2}}{\sqrt{U}}
$$

Where:

$\mathrm{f}_{\mathrm{e}}=0.14$ : the fraction of energy converted from pinch inductive energy into beam kinetic energy, $M$ is the mass number of ion, $Z_{\text {eff }}$ the effective charge, $b$ is the cathode radius, $r_{p}$ pinch radius, $I_{\text {pinch }}$ pinch current, The diode voltage U.

Energy in beam (Joul):

$$
E=Z_{\text {eff }} . U \times \text { ionsnumber }
$$

\section{Results and Discussion}

Plasma pinch properties which is the source of the ion beam in the UNU/ICTP PFF device

We use the followed parameters [15]:

a) Capacitor bank parameters: $\mathrm{L}_{0}=110 \mathrm{nH}, \mathrm{C}_{0}=30 \mu \mathrm{F}$, $\mathrm{r}_{0}=12 \mathrm{~m} \Omega$.

b) Parameters of the plasma tube: $\mathrm{b}=3.2 \mathrm{~cm}, \mathrm{a}=0.95 \mathrm{~cm}$, $\mathrm{z}_{0}=16 \mathrm{~cm}$.

c) Operating parameters: $\mathrm{V}_{0}=14 \mathrm{kV}$.

d) Parameters of the model: $\mathrm{f}_{\mathrm{m}}=0.08, \mathrm{f}_{\mathrm{c}}=0.7, \mathrm{f}_{\mathrm{mr}}=0.2$, $\mathrm{f}_{\mathrm{cr}}=0.7$

And then we use Lee code to carrying out many numerical experiments when helium gas pressure changed and we obtained the following results as shown in Table 1:

\begin{tabular}{|c|c|c|c|c|c|c|c|}
\hline Pressure & \multirow{3}{*}{ Fraction of energy $f_{\text {en }}$} & Ion & Effective Charge & Radius of Cathode b & Radius of Pinch $\mathbf{r}_{\mathbf{p}}$ & Current of Pinch $I_{\text {pinch }}$ & VoltageU \\
\hline \multirow{2}{*}{$\frac{P_{0}}{\text { (Torr) }}$} & & Mass & $\mathbf{Z}_{\text {eff }}$ & (cm) & (m) & (KA) & (V) \\
\hline & & \multicolumn{6}{|c|}{ Number } \\
\hline 1 & 0.14 & 4 & 2 & 3.2 & 0.13 & 102 & 99 \\
\hline 2 & 0.14 & 4 & 2 & 3.2 & 0.13 & 111 & 82 \\
\hline 3 & 0.14 & 4 & 2 & 3.2 & 0.131 & 114 & 70 \\
\hline 4 & 0.14 & 4 & 2 & 3.2 & 0.131 & 114 & 60 \\
\hline 5 & 0.14 & 4 & 2 & 3.2 & 0.131 & 113 & 52 \\
\hline 6 & 0.14 & 4 & 2 & 3.2 & 0.132 & 111 & 45 \\
\hline 7 & 0.14 & 4 & 2 & 3.2 & 0.132 & 108 & 38 \\
\hline 8 & 0.14 & 4 & 2 & 3.2 & 0.133 & 104 & 33 \\
\hline 9 & 0.14 & 4 & 2 & 3.2 & 0.134 & 100 & 28 \\
\hline 10 & 0.14 & 4 & 2 & 3.2 & 0.136 & 95 & 23 \\
\hline 11 & 0.14 & 4 & 2 & 3.2 & 0.138 & 91 & 19 \\
\hline 12 & 0.14 & 4 & 2 & 3.2 & 0.141 & 86 & 16 \\
\hline 13 & 0.14 & 4 & 2 & 3.2 & 0.146 & 80 & 12 \\
\hline 14 & 0.14 & 4 & 2 & 3.2 & 0.152 & 75 & 9 \\
\hline 15 & 0.14 & 4 & 2 & 3.2 & 0.158 & 69 & 7 \\
\hline
\end{tabular}

Table 1. Plasma pinch properties in UNU/ICTP PFF device.

Using the relations (1) and (2) we calculated the flux and energy of ions beam that emitted from UNU/ICTP PFF device when using helium gas as shown in Table 2:

Table 2. Flux and energy of helium ions beam in UNU/ ICTP PFF device.

\begin{tabular}{lll}
\hline Pressure & UNU/ICTP PFF & UNU/ICTP PFF \\
\hline Po & Flux of Ions & Beam Energy \\
\hline (Torr) & ${\left.\text { (Ions. } \mathbf{~ m}^{-2} \mathbf{s}^{-1}\right)}_{(J)}$ \\
\hline 1 & $8.59 \mathrm{E}+27$ & 7 \\
2 & $1.11 \mathrm{E}+28$ & 10 \\
3 & $1.26 \mathrm{E}+28$ & 11 \\
4 & $1.36 \mathrm{E}+28$ & 12 \\
5 & $1.42 \mathrm{E}+28$ & 13 \\
\hline
\end{tabular}

\begin{tabular}{lll}
\hline Pressure & UNU/ICTP PFF & UNU/ICTP PFF \\
\hline Po & Flux of Ions & Beam Energy \\
\hline (Torr) & $\left(\right.$ Ions. $\mathbf{~ m}^{-2} \mathbf{s}^{-1}$ ) & $(\mathbf{J})$ \\
\hline 6 & $1.45 \mathrm{E}+28$ & 12 \\
7 & $1.46 \mathrm{E}+28$ & 12 \\
8 & $1.46 \mathrm{E}+28$ & 12 \\
9 & $1.43 \mathrm{E}+28$ & 11 \\
10 & $1.39 \mathrm{E}+28$ & 10 \\
11 & $1.33 \mathrm{E}+28$ & 9 \\
12 & $1.25 \mathrm{E}+28$ & 8 \\
13 & $1.15 \mathrm{E}+28$ & 7 \\
14 & $1.03 \mathrm{E}+28$ & 6 \\
15 & $8.88 \mathrm{E}+27$ & 4 \\
\hline
\end{tabular}


Plasma pinch properties which is the source of the ion beam in the NX2 device:

We use the followed parameters [16]:

a) Capacitor bank parameters: $\mathrm{L}_{0}=20 \mathrm{nH}, \mathrm{C}_{0}=28 \mu \mathrm{F}, \mathrm{r}_{0}=2.3$ $\mathrm{m} \Omega$.

b) Parameters of the plasma tube: $b=4.1 \mathrm{~cm}, \mathrm{a}=1.9 \mathrm{~cm}$,

$$
\mathrm{z}_{0}=5 \mathrm{~cm} \text {. }
$$

c) Operating parameters: $\mathrm{V}_{0}=14 \mathrm{kV}$.

d) Parameters of the model: $\mathrm{f}_{\mathrm{m}}=0.08, \mathrm{f}_{\mathrm{c}}=0.7, \mathrm{f}_{\mathrm{mr}}=0.2, \mathrm{f}_{\mathrm{cr}}=0.7$

And then we use Lee code to carrying out many numerical experiments when helium gas pressure changed and we obtained the following results as shown in Table 3:

Table 3. Plasma pinch properties in NX2 device.

\begin{tabular}{|c|c|c|c|c|c|c|c|}
\hline Pressure & \multirow{3}{*}{ Fraction of energy $f_{\text {en }}$} & Ion & Effective Charge & Radius of Cathode b & Radius of Pinch $r_{p}$ & Current of Pinch $I_{\text {pinch }}$ & VoltageU \\
\hline \multirow{2}{*}{$\begin{array}{l}P_{0} \\
\text { (Torr) } \\
\end{array}$} & & Mass & $Z_{\text {eff }}$ & $(\mathrm{cm})$ & (m) & (KA) & (V) \\
\hline & & \multicolumn{6}{|c|}{ Number } \\
\hline 1 & 0.14 & 4 & 2 & 4.1 & 0.316 & 167 & 115 \\
\hline 2 & 0.14 & 4 & 2 & 4.1 & 0.317 & 190 & 105 \\
\hline 3 & 0.14 & 4 & 2 & 4.1 & 0.319 & 204 & 98 \\
\hline 4 & 0.14 & 4 & 2 & 4.1 & 0.321 & 212 & 92 \\
\hline 5 & 0.14 & 4 & 2 & 4.1 & 0.322 & 218 & 87 \\
\hline 6 & 0.14 & 4 & 2 & 4.1 & 0.324 & 222 & 82 \\
\hline 7 & 0.14 & 4 & 2 & 4.1 & 0.326 & 225 & 78 \\
\hline 8 & 0.14 & 4 & 2 & 4.1 & 0.327 & 227 & 74 \\
\hline 9 & 0.14 & 4 & 2 & 4.1 & 0.329 & 228 & 71 \\
\hline 10 & 0.14 & 4 & 2 & 4.1 & 0.331 & 229 & 67 \\
\hline 11 & 0.14 & 4 & 2 & 4.1 & 0.333 & 229 & 64 \\
\hline 12 & 0.14 & 4 & 2 & 4.1 & 0.335 & 229 & 61 \\
\hline 13 & 0.14 & 4 & 2 & 4.1 & 0.337 & 228 & 58 \\
\hline 14 & 0.14 & 4 & 2 & 4.1 & 0.34 & 227 & 55 \\
\hline 15 & 0.14 & 4 & 2 & 4.1 & 0.343 & 226 & 53 \\
\hline 16 & 0.14 & 4 & 2 & 4.1 & 0.345 & 225 & 50 \\
\hline 17 & 0.14 & 4 & 2 & 4.1 & 0.348 & 223 & 48 \\
\hline 18 & 0.14 & 4 & 2 & 4.1 & 0.352 & 221 & 46 \\
\hline 19 & 0.14 & 4 & 2 & 4.1 & 0.355 & 219 & 44 \\
\hline
\end{tabular}

Using the relations (1) and (2) we calculated the flux and energy of ions beam that emitted from NX2 device when using helium gas as shown in Table 4:

Table 4. Flux and energy of helium ions beam in NX2 device.

\begin{tabular}{lll}
\hline Pressure & NX2 & NX2 \\
\hline Po & Flux of Ions & Beam Energy \\
\hline (Torr) & ${\text { (Ions. } \mathbf{~ m}^{-2} \mathbf{s}^{-1} \text { ) }}^{\text {To }}$ \\
\hline 1 & $2.89 \mathrm{E}+27$ & 42 \\
2 & $3.87 \mathrm{E}+27$ & 64 \\
3 & $4.52 \mathrm{E}+27$ & 81 \\
4 & $5.00 \mathrm{E}+27$ & 94 \\
5 & $5.38 \mathrm{E}+27$ & 105 \\
6 & $5.68 \mathrm{E}+27$ & 114 \\
7 & $5.91 \mathrm{E}+27$ & 121 \\
8 & $6.09 \mathrm{E}+27$ & 127 \\
9 & $6.23 \mathrm{E}+27$ & 132 \\
10 & $6.33 \mathrm{E}+27$ & 136 \\
11 & $6.40 \mathrm{E}+27$ & 139 \\
12 & $6.44 \mathrm{E}+27$ & 141 \\
13 & $6.45 \mathrm{E}+27$ & 143 \\
14 & $6.44 \mathrm{E}+27$ & 144 \\
15 & $6.40 \mathrm{E}+27$ & 144 \\
16 & $6.35 \mathrm{E}+27$ & 144 \\
17 & $6.26 \mathrm{E}+27$ & 143 \\
18 & $6.16 \mathrm{E}+27$ & 142 \\
19 & $6.04 \mathrm{E}+27$ & 141 \\
\hline
\end{tabular}

Figure 1 shows the variations of ions beam flux with pressure changing in the studied devices:

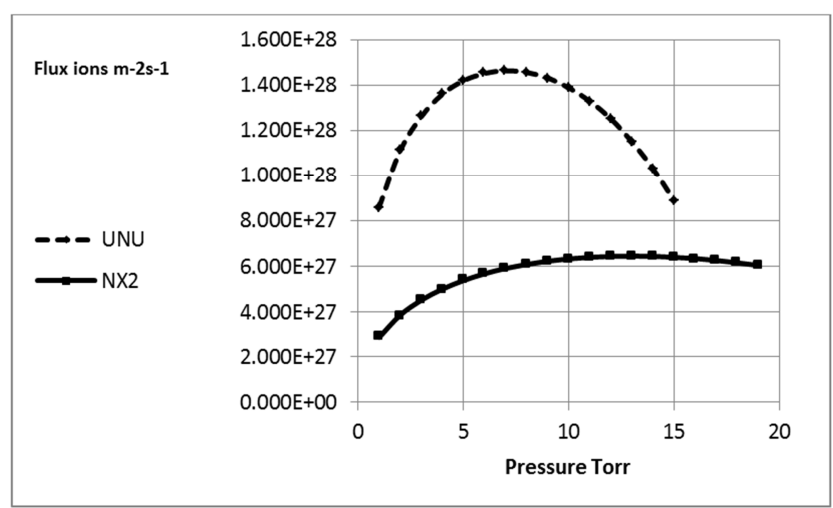

Figure 1. Changes the ions beam flux when the gas pressure changes in two devices.

Noted:

a) In both studied devices, the value of the ions beam flux increased with increasing the gas pressure until reaching a maximum value $1.463 \mathrm{E}+28$ ions. $\mathrm{m}^{-2} \mathrm{~s}^{-1}$ at 7 Torr in UNU/ ICTP PFF device while in NX2 device the maximum value is $6.442 \mathrm{E}+27$ ions. $\mathrm{m}^{-2} \mathrm{~s}^{-1}$ at 14 Torr and then decreasing while continuing pressure increasing. This is due to the behaviour of the pinch current $\left(\mathrm{I}_{\text {pinch }}\right)$ in both devices as shown in Tables 1 and 2 .

b) It is clear that the value of the ions beam flux in the UNU/ ICTP PFF is greater than that of the NX2 device. This can be explained by returning to relationship (1) and the results 
in Tables 1 and 2 where we note that the value of each fraction of the energy carried by the ions beam from the induced energy of the plasma pinch $\left(f_{e}\right)$ in both devices is constant at $(0.14)$ as well as the effective charge value $\left(\mathrm{Z}_{\mathrm{eff}}\right)$ and the helium ion mass number is constant. Thus, the increase in ions flux in the UNU/ ICTP PFF is due to the increase the ratio of the cathode radius to the pinch radius $\left(\mathrm{b} / \mathrm{r}_{\mathrm{p}}\right)$ and secondly the decrease in the value of the voltage within the formed plasma pinch since the flux of the ion beam is inversely proportional to this voltage.

Figure 2 shows the variations of ions beam energy with pressure changing in the studied devices:

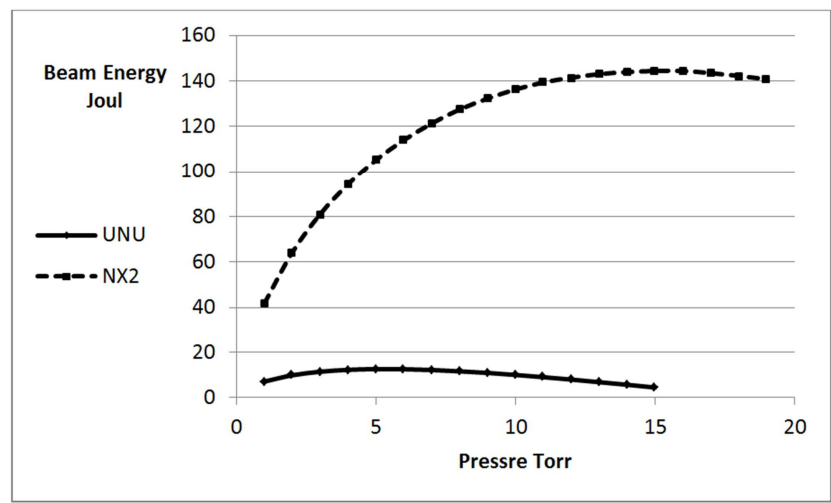

Figure 2. Changes the ions beam energy when the gas pressure changes in two devices.

We can see from Figure 2 that the energy of the ions beam from the NX2 device is higher. This can be explained by the relation (2) As follows:

The energy of the ions beam is directly proportional to both the induced voltage in plasma pinch and the number of emitted ions, this voltage is associated with two factor: first the value of the initial discharge current $\left(\mathrm{I}_{0}\right)$, which can be calculated from the relationship $I_{0}=\frac{V_{0}}{\sqrt{\frac{L_{0}}{C_{0}}}}$ and It is clear that because of low induction in NX2 then the initial discharge current is the highest, second the plasma pinch induction $\left(\mathrm{L}_{\mathrm{p}}\right)$ and because of the lower anode length in the NX2 compared to the UNU/ICTP PFF, the plasma layer reaches a higher speed simultaneously with the discharge current reaching its highest value, thus ensuring that the maximum value of capacitor bank power reaches to the pinch and thus increased the voltage within the plasma pinch, which contributes to the acquisition of helium ions after the collapse of the plasma pinch more energy, which explains the increase in the energy of ions beam which emitted by the device NX2, although they have a lower flux.

\section{Conclusions}

A series of numerical experiments were carried out using a Lee code to study the variations of flux and energy of the helium ions beam emitted by two dense plasma focus devices that are close to the operating energy and varying in geometry dimensions when the gas pressure changes.

The results showed that the flux in UNU/ICTP PFF device is higher than in NX2 device while in contrast the energy of the ion beam in the NX2 device was higher due to the difference in the value of the induced voltage within the pinch.

This difference in voltage value is due to the lower anode length in NX2 device compared to UNU/ICTP PFF device and this ensures that a larger amount of capacitor bank reaches to the formed plasma pinch and thus increases the value of the induced voltage within it.

\section{References}

[1] R. S. Rawat. (2017). Plasma Science and Technology for Emerging Economies. Springer Nature Singapore Pte Ltd. P 114.

[2] S. Lee, Radiative dense plasma focus computation package: RADPF. http://www. plasmafocus.net/IPFS/modelpackage/File1RADPF.htm.

[3] S. Lee, Plasma focus radiative model: review of the lee model code. J. Fusion Energy 33 (4), 319-335 (2014).

[4] M. Rosenbluth, in Magnetohydrodynamics, ed. by R. K. M. Landshoff (Stanford University Press, 1957), p. 57.

[5] D. Potter, The formation of high-density z-pinches. Nucl. Fusion 18 (6), 813-823 (1978).

[6] R. S. Rawat. (2017). Plasma Science and Technology for Emerging Economies. Springer Nature Singapore Pte Ltd. p123.

[7] M. Akel et al... (2014). Ion Beam Features Produced by Two Plasma Focus Machines Operated With Different Gases. IEEE TRANSACTIONS ON PLASMA SCIENCE, VOL. 42, NO. 9, SEPTEMBER 2014.

[8] M. Akel et al... (2014). Interaction of the high energy deuterons with the graphite target in the plasma focus devices based on Lee model. PHYSICS OF PLASMAS 21, 072507 (2014).

[9] S. M. Sadat Kiai et al... (2010). Design a $10 \mathrm{~kJ}$ IS Mather Type Plasma Focus for Solid Target Activation to Produce Short-Lived Radioisotopes 12C (d, n) 13N. J Fusion Energ (2010) 29: 421-426.

[10] B. Shirani. F. Abbasi (2013). Prospects for 13N Production in a Small Plasma Focus Device J Fusion Energ 32: 235-241.

[11] E. ANGELI et al... (2005.) PRODUCTION OF RADIOISOTOPES Within A Plasma Focus Device. Nuclear Technology \& Radiation Protection.

[12] BBie'nkowska (2004). APPLICATION OF THE ION BEAM EMITTED FROM PLASMA FOCUS DEVICE FOR TARGET ACTIVATION. acta physica slovaca vol. 54 No. 4 , 401. 407.

[13] Lee, S., \& Saw, S. H. (2013). Plasma focus ion beam fluence and flux-For various gases. PHYSICS OF PLASMAS 20, 062702 .

[14] Walid, S., \& Alaa, N. (2019). Ions Beam Properties Produced by NX2 Plasma Focus Device with Helium and Nitrogen Gas. American Journal of Modern Physics, 8 (1), 1-4. 
[15] M. Akel. S. Lee. (2012) Soft X-Ray Emission in the Water Window Region with Nitrogen Filling in a Low Energy Plasma Focus J Fusion Energ. DOI 10.1007/s10894-0129536-2.
[16] Lee, S., \& Saw, S. H. (2013). Scaling of Ion Beams from Plasma Focus in Various Gases. INTI International University Nilai Malaysia, 14 June 2013. 